\title{
Improving Selection for Resection of Synchronous Para-Aortic Lymph Node Metastases in Colorectal Cancer
}

\author{
Kazunosuke Yamada Shunsuke Tsukamoto Hiroki Ochiai Dai Shida \\ Yukihide Kanemitsu \\ Department of Colorectal Surgery, National Cancer Center Hospital, Tokyo, Japan
}

\section{Keywords}

Para-aortic lymph node metastasis - Colorectal cancer .

Para-aortic lymph node dissection

\begin{abstract}
Introduction: The clinical benefit of extended lymphadenectomy for synchronous extraregional lymph node metastasis, such as para-aortic lymph node (PALN) metastasis in colorectal cancer, remains highly controversial. Aim: To evaluate the clinical benefit of PALN dissection in colorectal cancer patients with synchronous PALN metastasis with or without multiorgan metastases. Methods: Thirty-six patients with pathologically positive PALN metastasis below the renal veins who underwent concurrent PALN dissection and primary colorectal cancer resection from January 1984 through September 2011 at the National Cancer Center Hospital in Tokyo, Japan, were included in this retrospective cohort study. We examined 5-year recurrence-free survival (RFS) rates in patient groups depending on the number of nodes involved ( $\leq 2$ and $\geq 3$ nodes) and on the presence or absence of other organ involvement (M1a and M1b,c categories in TNM staging). Results: The 5-year RFS rate was significantly different depending on the number of metastatic
\end{abstract}

\section{KARGER}

(c) 2018 S. Karger AG, Basel

E-Mail karger@karger.com

www.karger.com/dsu
PALNs (42.1 and $0.6 \%$ for PALN $\leq 2$ and $\geq 3$, respectively, $p=$ 0.01 ). The 5 -year RFS rate was significantly better in patients in the M1a category than in patients in the M1b and M1c categories ( 27.6 and $0.0 \%$, respectively, $p<0.01$ ). Twenty-nine patients (80.6\%) experienced recurrence after PALN dissection. Postoperative complications were seen in 14 (38.9\%) patients. Conclusion: PALN dissection below the renal veins for patients with isolated PALN metastasis with 2 or fewer involved PALNs may be effective in improving prognosis in colorectal cancer.

(c) 2018 S. Karger AG, Basel

\section{Background}

Approximately $12 \%$ of patients with colorectal cancer are diagnosed with stage IV cancer [1]. With recent advances in systemic chemotherapy and improved survival of patients with stage IV colorectal cancer [2,3], more aggressive hepatic or pulmonary resection has become a standard strategy to address liver or lung metastasis when R0 resection, or curative resection, can be achieved [4-7]. Moreover, when radical resection is technically possible, patients who undergo curative resection of distant metas- 
tases, regardless of the site, have longer survival than those who do not [8].

In contrast, the clinical benefit of extended lymphadenectomy for synchronous extraregional lymph node metastasis, such as para-aortic lymph node (PALN) metastasis, remains highly controversial. Generally, extraregional lymph node metastasis is considered a systemic disease not amenable to surgical cure.

PALNs are lymph nodes adjacent to the abdominal aorta. The incidence of isolated PALN metastasis is reported to be $1-2 \%$ [9]. Isolated PALN metastasis is categorized as M1 according to the TNM staging system of the Union for International Cancer Control [10]. To date, several case series have reported favorable outcomes in patients who underwent PALN dissection [11-14]; however, these reports examined only isolated PALN metastasis, and most studies were small and retrospective in nature.

The purpose of the current study was to evaluate the clinical benefit of PALN dissection in colorectal cancer with synchronous PALN metastasis with or without other organ metastases.

\section{Materials and Methods}

\section{Design}

This study was a retrospective cohort study.

\section{Patients}

Nine-hundred eighty-eight patients with stage IV colorectal cancer underwent surgery, including noncurative surgery, at the National Cancer Center Hospital, Tokyo, from January 1984 through September 2011. Of these patients, 52 underwent PALN dissection in addition to resection of primary colorectal cancer for suspected PALN metastasis. Generally, we consider combined PALN and primary colorectal cancer resection if the following criteria are met: (1) pathological diagnosis of colorectal cancer has been made; (2) PALN metastasis is suspected based on preoperative radiologic studies (computed tomography of abdomen/pelvis and/or positron emission tomography scan); (3) metastasis is suspected in PALN below the renal vein; and (4) the suspected PALN metastasis is amenable to complete resection. PALN was defined as located on the side of para-Aorta (black arrows) and distinguished from the lymph node at the origin of the inferior mesenteric artery (IMA), which was located in front of para-Aorta (Fig. 1). Therefore, in this study, the enlarged lymph nodes around the root of IMA were distinguished from PALN. Complete resection was defined as complete tumor resection with all margins being negative [15]. We excluded patients with secondary malignancies and with nodal metastasis above the renal veins. Thirty-six patients who were pathologically positive for PALN metastasis were included in the study. PALN dissection was performed in the area with the following boundaries: renal hilum, bifurcation of the iliac artery,

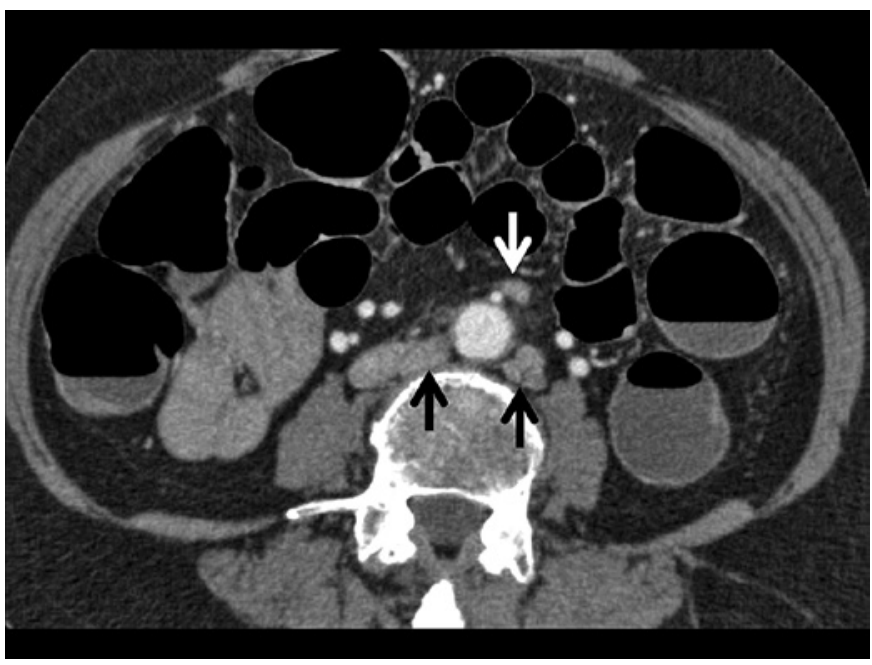

Fig. 1. Para-aortic lymph nodes (black arrows) and a lymph node at the origin of the inferior mesenteric artery (white arrow).

bilateral ureters, and iliopsoas muscle. Survival statuses and pathological data were obtained retrospectively from medical records.

\section{Main Outcome Measures}

The primary end points were 5-year overall survival (OS) and recurrence-free survival (RFS) rates.

\section{Pathological Examination of Lymph Nodes}

In the Japanese Classification of Colorectal Carcinoma [16], regional lymph nodes are classified into 3 groups according to their location: pericolic/perirectal nodes are situated close to the bowel wall, intermediate nodes lie along the feeding arteries, and main nodes are located at the origin of the feeding artery. In addition, lateral pelvic nodes lie along the common internal and external iliac vessels and downward to the level of the obturator internus muscle. Immediately after resection, the surgeons who performed the operation also dissected all regional lymph nodes individually from the adipose connective tissue of the specimen. Nodes were assigned to the appropriate station according to the classification of the Japanese Society for Cancer of the Colon and Rectum [16].

\section{Statistical Analysis}

Statistical analysis was carried out with SPSS for Windows (version 22.0, SPSS Inc., Chicago, IL, USA) software. Survival curves were estimated by the Kaplan-Meier method, and comparisons between curves were made by the log-rank test. A probability level of $p<0.05$ was used for statistical significance.

\section{Results}

Patient characteristics are summarized in Table 1. The median age was 57.0 (range 46.3-65.8) years. Fifteen $(42.7 \%)$ patients were women and 21 (58.3\%) were men. 
Tumor location was categorized based on the following definition: the right colon was defined as the cecum, ascending colon, and transverse colon, and the left colon was defined as the splenic flexure, descending colon, and sigmoid colon. Four (11.1\%) tumors were located in the right colon, 13 (36.1\%) in the left colon, and 19 (52.8\%) in the rectum. In terms of surgical procedure type, 9 (25.0\%) patients underwent abdominoperineal resection (APR), 8 (22.2\%) sigmoidectomy, 7 (19.4\%) low anterior resection, $6(16.7 \%)$ high anterior resection, 4 (11.1\%) right hemicolectomy, 1 (2.8\%) left hemicolectomy, and 1 $(2.8 \%)$ total pelvic exenteration. The median operating time was $340(140-760) \mathrm{min}$ and the median estimated blood loss was 1,095 $(42-4,315) \mathrm{g}$. The median hospital stay duration was 24.5 (14-429) days.

Pathological characteristics are summarized in Table 2 . The moderately differentiated type (52.8\%) was the most common histological type, followed by well-differentiated (22.2\%), mucinous (13.9\%), signet ring cell (5.6\%), and poorly differentiated (5.6\%). There were 11 (30.6\%) T3 tumors, 18 (50.0\%) T4a tumors, and 7 (19.4\%) T4b tumors. All patients had nodal metastasis at the pericolic/perirectal nodes. However, only 23 (63.9\%) patients had nodal metastasis at the origin of the arteries. Seven (19.4\%) patients did not have nodal metastasis around the major arteries or in a lateral pelvic node; that is, they demonstrated skip metastases to PALN. In the past study, it was reported that the existence of 2 or less PALN metastases is associated with a good prognosis [13], and in this study, PALN metastasis was classified into 2 groups ( $\leq 2$ or $\geq 3$ metastatic PALNs). The median number of lymph node retrieved was 36 (8-99). The median lymph node ratio (LNR), which is the ratio of lymph nodes metastasis to dissected nodes, was $0.35(0.02-0.98)$. The median LNR in $\geq 3$ PALN metastasis was significantly larger than in $\leq 2$ PALN metastasis $(0.55$ [0.26-0.98] vs. 0.20 [0.020.5 ], $p \leq 0.01)$. No lymph node metastasis was observed at the origin of the inferior mesenteric artery in 2 or less PALN metastases.

In terms of other organ involvement, 29 (80.6\%) patients were in category M1a (only PALN metastasis), 5 (13.9\%) patients were in category M1b (PALN and liver metastases), and 2 (5.6\%) patients were in category M1c (PALN and peritoneal metastases) of the TNM staging system. Two (5.6\%) patients received neoadjuvant chemotherapy and $25(69.4 \%)$ patients received adjuvant chemotherapy, while $11(30.1 \%)$ patients received no chemotherapy.

Postoperative complications were observed in 14 (38.9\%) patients (Table 3). These complications were
Table 1. Patient characteristics

\begin{tabular}{lc}
\hline & $n=36$ \\
\hline Age* & $57.0(46.3-65.8)$ \\
Gender, $n(\%)$ & $21(58.3)$ \\
$\quad$ Male & $15(42.7)$ \\
Female & \\
Location, $n(\%)$ & $4(11.1)$ \\
Right colon & $13(36.1)$ \\
Left colon & $19(52.8)$ \\
Rectum & \\
Surgical procedure, $n(\%)$ & $9(25.0)$ \\
APR & $8(22.2)$ \\
Sigmoidectomy & $7(19.4)$ \\
LAR & $6(16.7)$ \\
HAR & $4(11.1)$ \\
Right hemicolectomy & $1(2.8)$ \\
Left hemicolectomy & $1(2.8)$ \\
TPE & $340(140-760)$ \\
Operation time, min* & $1,095(42-4,315)$ \\
Blood loss, g* & $24.5(14-429)$ \\
Hospital stay, days* &
\end{tabular}

* Values are median (range).

APR, abdominoperineal resection; LAR, low anterior resection; HAR, high anterior resection; TPE, total pelvic exenteration.

Table 2. Pathological characteristics

$$
n=36
$$

Histology, $n$ (\%)

Moderately differentiated adenocarcinoma 19 (52.8)

Well-differentiated adenocarcinoma $\quad 8(22.2)$

Mucinous adenocarcinoma $\quad 5(13.9)$

Signet ring cell carcinoma 2 (5.6)

Poorly differentiated adenocarcinoma 2 (5.6)

$\mathrm{T}, n(\%)$

pT3 $11(30.6)$

pT4a $18(50.0)$

pT4b

$7(19.4)$

Extent of lymph node metastasis, $n(\%)$

Pericolic/perirectal $36(100.0)$

Intermediate $29(80.6)$

Main 23 (63.9)

Lateral pelvic node $\quad 13(36.1)$

$\mathrm{M}, n(\%)$

pM1a (PALN only) $29(80.5)$

pM1b (PALN + liver metastasis) 5 (13.9)

pM1c (PALN + peritoneal metastasis) $2(5.6)$

Number of PALN metastases* $\quad 2.0(1.0-24.0)$

* Values are median (range).

PALN, para-aortic lymph node. 
classified according to the Clavien and Dindo classification [17]. Three (8.3\%) patients had grade I (urinary retention, ileus, and surgical site infection), 5 (13.9\%) patients had grade II ( 2 surgical site infection, 2 urinary tract infection, 1 urinary retention, 1 diarrhea, 1 acute compartment syndrome, 1 deep vein thrombosis), 1 (2.8\%) patient had grade IIIa (intra-abdominal abscess), 2 (5.6\%) patients had grade IIIb (intra-abdominal abscess, chylous ascites), and 0 patients had grade IV and V complications. There was no perioperative death.

Median follow-up of all cases was 2.1 (range 0.9-5.2) years. Median follow-up of patients who survived past 5 years after surgery was 11.8 (range 7.0-22) years, and that of patients who died within 5 years of surgery was 1.6 (range $0-5.0$ ) years. The 5-year OS rate was $25 \%$ and 5 -year RFS rate was $22.2 \%$ (Fig. 2a, b). The 5 -year RFS rate RFS was not significantly different in both groups in the colon and rectum (Fig. 3a). The 5-year RFS rate differed significantly depending on whether there were $\leq 2$ or $\geq 3$ metastatic PALNs (42.1 and $0.6 \%$, respectively, $p=0.01$; Fig. $3 b$ ). In addition, the 5 -year RFS rate was significantly better in patients in category M1a as opposed to category M1b or M1c (27.6 and $0.0 \%$, respectively, $p<0.01$; Fig. $3 c$ ).

Twenty-nine patients $(80.6 \%)$ experienced recurrence after PALN dissection. The most common site of recurrence was the lung (29.0\%), followed by local recurrence (26.0\%), distant LN (15.0\%), bone (12.0\%), liver (9.0\%), peritoneum (6.0\%), and brain (3.0\%).

\section{Discussion}

PALN dissection was first described in 1959 as a modification of the Miles APR in the management of rectal cancer [18]. By prophylactically removing lymph nodebearing tissues from the para-aortic and vena caval regions between the ureters from the duodenum down to the levator ani muscles, PALN dissection aimed to reduce local recurrence rates, which was common despite APR. However, routine PALN dissection has since been abandoned by most institutions in view of the increased surgical morbidity without corresponding improvements in recurrence rates and OS [19]. In the last decade, PALN dissection has been adopted mainly for curative purposes in PALN metastasis.

Very few studies have been performed on PALN dissection in primary colon cancer. Ogura et al. [20] reported that only $0.67 \%$ of patients with primary colorectal cancer who underwent surgery had isolated PALN metastasis. In 2 case series [21,22], which included one patient
Table 3. Postoperative complications based on the Clavien and Dindo classification

\begin{tabular}{lc}
\hline Complications & $(n=36)$ \\
\hline No complication, $n(\%)$ & $22(61.1)$ \\
Grade I, $n(\%)$ & $1(2.8)$ \\
$\quad$ Urinary retention & $1(2.8)$ \\
$\quad$ Ileus & $1(2.8)$ \\
SSI & \\
Grade II, $n$ (\%) & $2(5.6)$ \\
$\quad$ SSI & $2(5.6)$ \\
UTI & $1(2.8)$ \\
$\quad$ Urinary retention & $1(2.8)$ \\
$\quad$ Diarrhea & $1(2.8)$ \\
Acute compartment syndrome & $1(2.8)$ \\
DVT & $1(2.8)$ \\
Grade IIIa, $n$ (\%) & \\
Intra-abdominal abscess & $1(2.8)$ \\
Grade IIIb, $n$ (\%) & $1(2.8)$ \\
$\quad$ Intra-abdominal abscess & Nil \\
Chylous ascites & Nil \\
Grade IV & \\
Grade V &
\end{tabular}

SSI, surgical site infection; UTI, urinary tract infection; DVT, deep vein thrombosis.

with synchronous isolated PALM and the other with no other organ metastasis, the 5-year OS ranged from 22.7 to $33.9 \%$ and the 5 -year disease free survival from 17.6 to $26.5 \%$. In our study, the 5-year OS rate and the 5-year RFS rate were 25.0 and $22.2 \%$, respectively, consistent with previous reports. In addition, our report also included cases of liver metastasis and peritoneal dissemination, which are expected to lower OS and RFS rates. Choi et al. [13] reported that the presence of 2 or fewer PALN metastases was associated with a good prognosis, suggesting that the presence of 2 or fewer PALN metastases may be a good indication for PALN dissection. Several other reports indicate that the number of PALN metastases may be a prognostic factor $[20,22,23]$. Likewise, we found that the 5-year RFS rate differed significantly depending on the number of PALN metastasis ( $\leq 2$ or $\geq 3$ ). These results may suggest that patients with 3 or more PALN metastases should not undergo PALN dissection. On the other hand, tumor location did not affect prognosis. However, it might have no significant difference due to a small sample size.

The surgical approach to colorectal cancer patients with multiorgan metastases, including PALN, remains controversial, with few studies reporting on this topic. Recently, several centers have reported on resection of 
Fig. 2. a Kaplan-Meier overall survival curve for all patients. b Kaplan-Meier curve for recurrence-free survival for all patients.

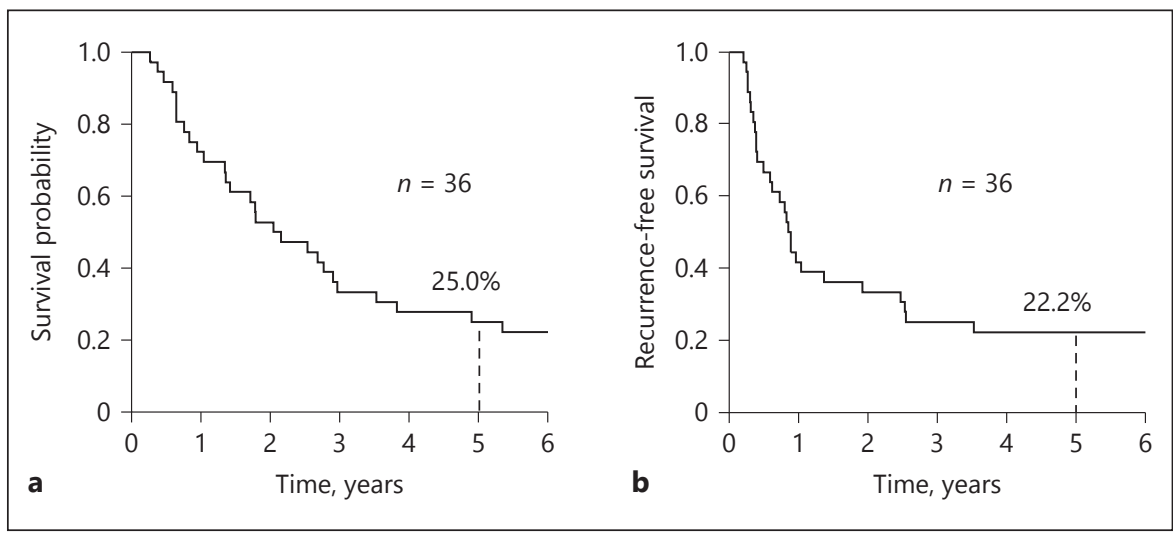

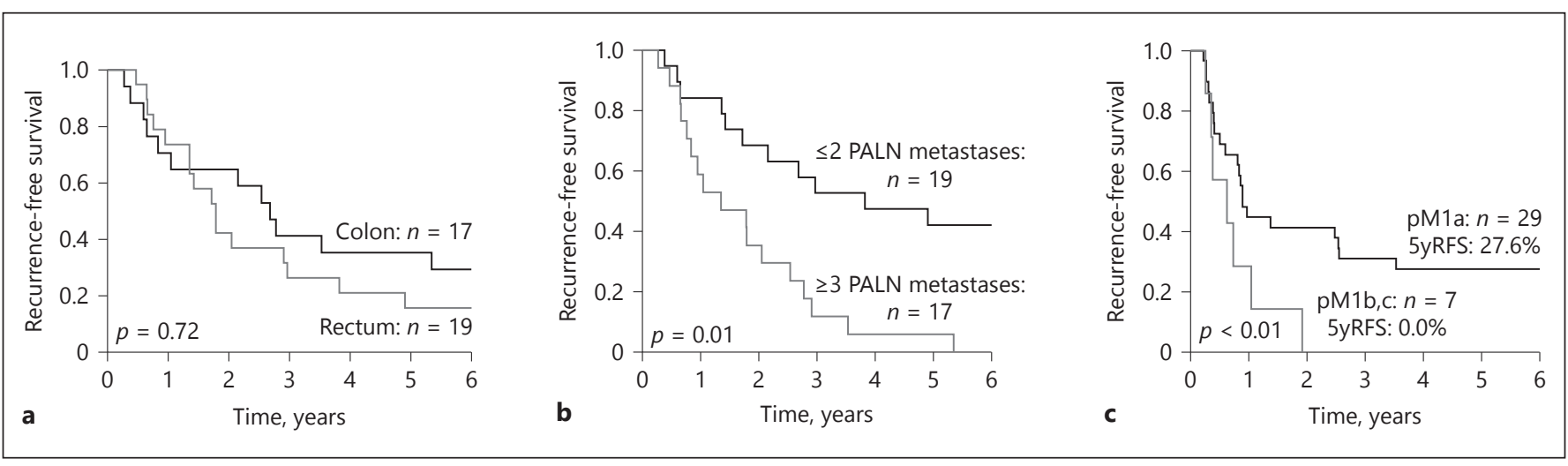

Fig. 3. a Kaplan-Meier recurrence-free survival curves for all patients comparing tumor location. b Kaplan-Meier curves for recurrence-free survival for patients with $\leq 2$ vs. $\geq 3$ PALN metasta- ses. c Kaplan-Meier curves for recurrence-free survival for patients with category pM1a vs pM1b,c. PALN, para-aortic lymph node. synchronous or metachronous pulmonary metastases in the setting of colorectal liver metastasis and concluded that resection of hepatic and extrahepatic disease in wellselected patients may provide the possibility of long-term survival [24-27]. However, Pulitano et al. [26] reported that colorectal cancer patients with PALN metastasis and liver metastasis had particularly poor survival (median survival, 13 months; $n=14$ ). Interestingly, in our report, the 5-year RFS rate was significantly better in the M1a group than that in the M1b and M1c group (27.6 and $0.0 \%$, respectively, $p<0.01)$. These results suggest that patients with PALN metastasis with other organ involvement may not benefit from PALN dissection.

In terms of surgical complications in PALN dissection, according to a systematic review by Wong et al. [28], its incidence ranged from 7.8 to $33 \%$, and were mainly lowgrade in nature. In the current study, postoperative complications were seen in 14 (38.9\%) patients, with only 8.3\% experiencing Clavien and Dindo classification grade
III or above, with no perioperative death. These results suggest that the safety of PALN dissection is reassuring.

Recurrence following curative treatment for PALN metastasis is common, with 56 to $80 \%$ of patients experiencing either local or distant recurrences after PALN dissection for colorectal cancer [28]. In our study, $80.6 \%$ of patients experienced recurrence after PALN dissection and distant metastasis was the predominant pattern. The efficacy and safety of adjuvant chemotherapy following the resection of distant metastasis in colorectal cancer have not been established, and no randomized controlled trials have examined whether or not it extends survival $[29,30]$. Nevertheless, with such high recurrence rates, it is evident that colorectal cancer patients with PALN metastasis have a poor prognosis, and consideration of adjuvant chemotherapy is therefore important.

In general, preoperative diagnosis of PALN metastasis was difficult and the positive predictive value of the preoperative radiologic investigations for the diagnosis 
of PALN metastasis was $69.2 \%$ in our study. Min et al. [21] also reported a positive predictive value of $47.4 \%$. Moreover, intraoperative assessment for the presence of lymph node metastases is not reliable [31]. Therefore, only our results cannot be used to decide on PALN dissection. However, in a recent report, the accuracies of nodal status prediction from combination of MRI and PET/CT in rectal cancer revealed a sensitivity of $94 \%$, a specificity of $83 \%$, and an accuracy of $90 \%$ [32]. Besides, the LNR, which has been shown to be a powerful prognostic factor in several solid cancer types [33-35], in $\geq 3$ PALN metastasis was significantly larger than in $\leq 2$ PALN metastasis in our study. Consequently, in case of multiple mesenteric lymph node metastases are indicated and 3 or more PALN metastasis is suspected in preoperative diagnosis, systemic chemotherapy may be considered.

Only 2 patients underwent preoperative chemotherapy in our study. Evidence supporting resection of extrahepatic metastases in patients with metastatic colorectal cancer is limited. National Comprehensive Cancer Network recommends that a course of an active systemic therapy regimen for metastatic disease and can be given before, between, or after resections [36]. On the other hand, Japanese Society for Cancer of the Colon and Rectum guidelines do not mention the treatment policy because the efficacy and safety of perioperative chemotherapy for distant metastatic lesion in cases of colorectal cancer have not yet been established [37]. Our facility had been actively selecting surgery for these patients. For this reason, these patients who received upfront chemotherapy were considered to be poor prognostic groups, and few cases had to undergo surgery.

The current study focused on synchronous PALN metastasis in colorectal cancer because metachronous PALN metastasis may have different clinical characteristics. National Comprehensive Cancer Network guidelines indicate that metastatic colorectal liver disease that develops metachronously is associated with a less disseminated disease state and a better prognosis than synchronous metastatic colorectal liver disease. Moreover, for patients who have resectable metastatic disease, treatment is resection with 6 months of perioperative chemotherapy (pre- or postoperative or a combination of both), with choice of regimens based on previous therapy [36]. A similar biological behavior is expected in PALN metastasis, but further studies in order to provide evidence supporting resection of metachronous PALN metastasis are needed.

\section{Limitations}

The current study had several limitations, including a small sample size and selection bias. It was difficult to demonstrate directly whether or not PALN dissection itself contributed to the improved prognosis. Patients who underwent systemic chemotherapy without surgery were excluded from the control group, and the perioperative strategy was not consistent. However, our results clearly showed that long-term survival could be expected in select patients with isolated PALN metastasis, with only one or 2 nodes involved, in colorectal cancer. In light of our results supporting the role of PALN dissection, randomized controlled trials are required to determine selection criteria and the optimal course of management for PALN metastasis.

\section{Conclusion}

In conclusion, our study suggests that PALN dissection for patients with 2 or fewer PALN metastases without other organ involvement in colorectal cancer may be effective in improving the prognosis. Randomized controlled trials are required to assess the efficacy of PALN dissection versus systemic chemotherapy in patients with clinically suspected PALN metastasis.

\section{Disclosure Statement}

The authors confirm that there are no conflicts of interest.

References

1 Howlader N NA, Krapcho M, Miller D, Bishop K, Kosary CL, Yu M, Ruhl J, Tatalovich Z, Mariotto A, Lewis DR, Chen HS, Feuer EJ, Cronin KA: SEER Cancer Statistics Review (CSR) 1975-2014. National Cancer Institute. https://seer.cancer.gov/csr/1975_2014 (accessed on January 11, 2018).

2 Goldberg RM, Sargent DJ, Morton RF, Fuchs CS, Ramanathan RK, Williamson SK, Findlay BP, Pitot HC, Alberts SR: A randomized controlled trial of fluorouracil plus leucovorin, irinotecan, and oxaliplatin combinations in patients with previously untreated metastatic colorectal cancer. J Clin Oncol 2004;22:2330 .

3 Tournigand C, Andre T, Achille E, Lledo G, Flesh M, Mery-Mignard D, Quinaux E, Couteau C, Buyse M, Ganem G, Landi B, Colin P, Louvet C, de Gramont A: FOLFIRI followed by FOLFOX6 or the reverse sequence in advanced colorectal cancer: a randomized GERCOR study. J Clin Oncol 2004;22:229-237. 
4 Ike H, Shimada H, Ohki S, Togo S, Yamaguchi S, Ichikawa Y: Results of aggressive resection of lung metastases from colorectal carcinoma detected by intensive follow-up. Dis Colon Rectum 2002;45:468-473.

5 Pfannschmidt J, Hoffmann H, Dienemann $\mathrm{H}$ : Reported outcome factors for pulmonary resection in metastatic colorectal cancer. J Thorac Oncol 2010;5(6 suppl 2):S172-S178.

6 Saiura A, Yamamoto J, Hasegawa K, Koga R, Sakamoto Y, Hata S, Makuuchi M, Kokudo N: Liver resection for multiple colorectal liver metastases with surgery up-front approach: bi-institutional analysis of 736 consecutive cases. World J Surg 2012;36:2171-2178.

7 Saiura A, Yamamoto J, Koga R, Takahashi Y, Takahashi M, Inoue Y, Ono Y, Kokudo N: Favorable outcome after repeat resection for colorectal liver metastases. Ann Surg Oncol 2014;21:4293-4299.

8 Mahmoud N, Bullard Dunn K: Metastasectomy for stage IV colorectal cancer. Dis Colon Rectum 2010;53:1080-1092.

9 Gagniere J, Dupre A, Chabaud S, Peyrat P, Meeus P, Rivoire M: Retroperitoneal nodal metastases from colorectal cancer: curable metastases with radical retroperitoneal lymphadenectomy in selected patients. Eur J Surg Oncol 2015;41:731-737.

10 UICC: TNM Classification of Malignant Tumours (ed 8). New York, John Wiley \& Sons, 2017.

11 Shibata D, Paty PB, Guillem JG, Wong WD, Cohen AM: Surgical management of isolated retroperitoneal recurrences of colorectal carcinoma. Dis Colon Rectum 2002;45:795-801.

12 Min BS, Kim NK, Sohn SK, Cho CH, Lee KY, Baik SH: Isolated paraaortic lymph-node recurrence after the curative resection of colorectal carcinoma. J Surg Oncol 2008;97:136-140.

13 Choi PW, Kim HC, Kim AY, Jung SH, Yu CS, Kim JC: Extensive lymphadenectomy in colorectal cancer with isolated para-aortic lymph node metastasis below the level of renal vessels. J Surg Oncol 2010;101:66-71.

14 Lee J, Chang JS, Shin SJ, Lim JS, Keum KC, Kim NK, Ahn JB, Kim TI, Koom WS: Incorporation of radiotherapy in the multidisciplinary treatment of isolated retroperitoneal lymph node recurrence from colorectal cancer. Ann Surg Oncol 2015;22:1520-1526.

15 Amin MB, Edge SB, Greene FL, et al: AJCC Cancer Staging Manual, ed 8. Chicago, Springer, 2017.

16 Japanese Classification of Colorectal Carcinoma, Second English Edition. Tokyo, Kanehara \& Co., Ltd., 2009.

17 Dindo D, Demartines N, Clavien PA: Classification of surgical complications: a new proposal with evaluation in a cohort of $6336 \mathrm{pa}-$ tients and results of a survey. Ann Surg 2004; 240:205-213.

18 Stearns MW Jr, Deddish MR: Five-year results of abdominopelvic lymph node dissec- tion for carcinoma of the rectum. Dis Colon Rectum 1959;2:169-172.

19 Leggeri A, Roseano M, Balani A, Turoldo A: Lumboaortic and iliac lymphadenectomy: what is the role today? Dis Colon Rectum 1994;37(suppl 2):S54-S61.

20 Ogura A, Akiyoshi T, Takatsu Y, Nagata J, Nagasaki T, Konishi T, Fujimoto Y, Nagayama S, Fukunaga Y, Ueno M: The significance of extended lymphadenectomy for colorectal cancer with isolated synchronous extraregional lymph node metastasis. Asian J Surg 2017;40:254-261.

21 Min BS, Kim JS, Kim NK, Lim JS, Lee KY, Cho $\mathrm{CH}$, Sohn SK: Extended lymph node dissection for rectal cancer with radiologically diagnosed extramesenteric lymph node metastasis. Ann Surg Oncol 2009;16:3271-3278.

22 Bae SU, Hur H, Min BS, Baik SH, Lee KY, Kim NK: Which patients with isolated para-aortic lymph node metastasis will truly benefit from extended lymph node dissection for colon cancer? Cancer Res Treat 2017, Epub ahead of print.

23 Song SH, Park SY, Park JS, Kim HJ, Yang CS, Choi GS: Laparoscopic para-aortic lymph node dissection for patients with primary colorectal cancer and clinically suspected para-aortic lymph nodes. Ann Surg Treat Res 2016;90:29-35.

24 Shah SA, Haddad R, Al-Sukhni W, Kim RD, Greig PD, Grant DR, Taylor BR, Langer B, Gallinger S, Wei AC: Surgical resection of hepatic and pulmonary metastases from colorectal carcinoma. J Am Coll Surg 2006; 202:468-475.

25 Mise Y, Imamura H, Hashimoto T, Seyama Y, Aoki T, Hasegawa K, Beck Y, Sugawara Y, Makuuchi M, Nakajima J, Kokudo N: Cohort study of the survival benefit of resection for recurrent hepatic and/or pulmonary metastases after primary hepatectomy for colorectal metastases. Ann Surg 2010;251:902-909.

26 Pulitano C, Bodingbauer M, Aldrighetti L, de Jong MC, Castillo F, Schulick RD, Parks RW, Choti MA, Wigmore SJ, Gruenberger T, Pawlik TM: Liver resection for colorectal metastases in presence of extrahepatic disease: results from an international multi-institutional analysis. Ann Surg Oncol 2011;18:1380-1388.

27 Tsukamoto S, Kinugasa Y, Yamaguchi T, Shiomi A: Survival after resection of liver and lung colorectal metastases in the era of modern multidisciplinary therapy. Int J Colorectal Dis 2014;29:81-87.

28 Wong JS, Tan GH, Teo MC: Management of para-aortic lymph node metastasis in colorectal patients: a systemic review. Surg Oncol 2016;25:411-418.

29 Portier G, Elias D, Bouche O, Rougier P, Bosset JF, Saric J, Belghiti J, Piedbois P, Guimbaud R, Nordlinger B, Bugat R, Lazorthes F, Bedenne L: Multicenter randomized trial of adjuvant fluorouracil and folinic acid com- pared with surgery alone after resection of colorectal liver metastases: FFCD ACHBTH AURC 9002 trial. J Clin Oncol 2006;24:49764982.

30 Mitry E, Fields AL, Bleiberg H, Labianca R, Portier G, Tu D, Nitti D, Torri V, Elias D, O'Callaghan C, Langer B, Martignoni G, Bouche O, Lazorthes F, Van Cutsem E, Bedenne L, Moore MJ, Rougier P: Adjuvant chemotherapy after potentially curative resection of metastases from colorectal cancer: a pooled analysis of two randomized trials. J Clin Oncol 2008;26:4906-4911.

31 Kanemitsu Y, Hirai T, Komori K, Kato T: Survival benefit of high ligation of the inferior mesenteric artery in sigmoid colon or rectal cancer surgery. Br J Surg 2006;93:609-615.

32 Kim DJ, Kim JH, Ryu YH, Jeon TJ, Yu JS, Chung JJ: Nodal staging of rectal cancer: highresolution pelvic MRI versus ${ }^{18} \mathrm{~F}$-FDGPET/ CT. J Comput Assist Tomogr 2011;35:531534.

33 Inoue K, Nakane Y, Iiyama H, Sato M, Kanbara T, Nakai K, Okumura S, Yamamichi K, Hioki K: The superiority of ratio-based lymph node staging in gastric carcinoma. Ann Surg Oncol 2002;9:27-34.

34 Woodward WA, Vinh-Hung V, Ueno NT, Cheng YC, Royce M, Tai P, Vlastos G, Wallace AM, Hortobagyi GN, Nieto Y: Prognostic value of nodal ratios in node-positive breast cancer. J Clin Oncol 2006;24:2910-2916.

35 Xing Y, Badgwell BD, Ross MI, Gershenwald JE, Lee JE, Mansfield PF, Lucci A, Cormier JN: Lymph node ratio predicts disease-specific survival in melanoma patients. Cancer 2009; 115:2505-2513.

36 Benson AB 3rd, Venook AP, Cederquist L, Chan E, Chen YJ, Cooper HS, Deming D, Engstrom PF, Enzinger PC, Fichera A, Grem JL, Grothey A, Hochster HS, Hoffe S, Hunt S, Kamel A, Kirilcuk N, Krishnamurthi S, Messersmith WA, Mulcahy MF, Murphy JD, Nurkin S, Saltz L, Sharma S, Shibata D, Skibber JM, Sofocleous CT, Stoffel EM, StotskyHimelfarb E, Willett CG, Wu CS, Gregory KM, Freedman-Cass D: Colon Cancer, Version 1.2017, NCCN Clinical Practice Guidelines in Oncology. J Natl Compr Canc Netw 2017;15:370-398.

37 Watanabe T, Muro K, Ajioka Y, Hashiguchi $\mathrm{Y}$, Ito $\mathrm{Y}$, Saito $\mathrm{Y}$, Hamaguchi $\mathrm{T}$, Ishida $\mathrm{H}$, Ishiguro M, Ishihara S, Kanemitsu Y, Kawano H, Kinugasa Y, Kokudo N, Murofushi K, Nakajima T, Oka S, Sakai Y, Tsuji A, Uehara K, Ueno H, Yamazaki K, Yoshida M, Yoshino T, Boku N, Fujimori T, Itabashi M, Koinuma N, Morita T, Nishimura G, Sakata Y, Shimada Y, Takahashi K, Tanaka S, Tsuruta O, Yamaguchi T, Yamaguchi N, Tanaka T, Kotake K, Sugihara K: Japanese Society for Cancer of the Colon and Rectum (JSCCR) guidelines 2016 for the treatment of colorectal cancer. Int $J$ Clin Oncol 2018;23:1-34.
Improving Selection for Resection of Synchronous PALN Metastases 